\title{
PREDIÇÃO DE TOXICIDADE DOS ESTABILIZANTES USUAIS EM PROPELENTES À BASE DE NITROCELULOSE E DE SEUS PRINCIPAIS PRODUTOS DE DEGRADAÇÃO
}

\author{
Rodrigo L. B. Rodrigues ${ }^{\mathrm{a}}$, Jakler Nichele, Tanos C. C. França, ${ }^{\mathrm{a}, \mathrm{b}, *}$ e Letivan G. Mendonça Filho ${ }^{\mathrm{a}, \#}$ \\ ${ }^{a}$ Seção de Engenharia Química, Instituto Militar de Engenharia, 22290-270 Rio de Janeiro - RJ, Brasil \\ ${ }^{b}$ Faculty of Informatics and Management, Center for Basic and Applied Research, University of Hradec Králové, Rokitanskeho, \\ 62, 50003 Hradec Kralove, Czech Republic
}

Recebido em 27/02/2018; aceito em 13/06/2018; publicado na web em 23/07/2018

\begin{abstract}
PREDICTION OF TOXICITY OF THE USUAL STABILIZERS IN NITROCELLULOSE BASED PROPELLANTS AND THEIR MAIN DEGRADATION PRODUCTS. This work aims to establish a hierarchy of risks of the stabilizers used in nitrocellulose-based propellants concerning to toxicity, carcinogenicity and mutagenicity. Four stabilizers - diphenylamine, ethyl centralite, akardite-II and 2-nitrodiphenylamine - and their products of degradation were investigated. The prediction of the toxicity was based on the analysis of their structures with the software Lazar, using the OpenTox framework. Our results provide a quantitative reference of the risks involved in the manufacturing and disposal of propellants due to the use of such stabilizers.
\end{abstract}

Keywords: propellants; Lazar software; toxicity; carcinogenicity; mutagenicity.

\section{INTRODUÇÃO}

Propelentes são substâncias químicas puras ou misturas utilizadas com a finalidade de mover objetos. Podem ser gases, líquidos ou sólidos e sua ação pode ou não envolver uma reação química. Exemplos de propelentes são o hidrogênio, o hélio, a gasolina, combustíveis de foguetes e as pólvoras utilizadas em munições. Estas últimas geralmente contêm a nitrocelulose (NC) como principal componente de suas formulações, cuja instabilidade demanda a utilização de estabilizantes. ${ }^{1}$

Os estabilizantes são substâncias utilizadas nas formulações de propelentes com a finalidade de retardar o processo de degradação natural da NC, prolongando o tempo de vida dos propelentes à base de NC, quando em condições adequadas de armazenamento. ${ }^{1}$ No processo de interação química entre os estabilizantes e os vapores nitrosos oriundos da degradação da NC, é gerada uma grande variedade de compostos nitrosados, dentre os quais se destacam as $\mathrm{N}$-nitroso-aminas e as N-nitroso-amidas. Tais substâncias tóxicas são as principais responsáveis pelos riscos à saúde dos profissionais que manipulam pólvoras e propelentes, assim como, pela contaminação do ambiente. Há relatos na literatura sobre o risco desses compostos serem carcinogênicos, mutagênicos e prejudiciais à reprodução (abreviados aqui como CMR). ${ }^{1-5}$

Os estabilizantes utilizados comercialmente hoje em dia na composição de propelentes a base de NC são todos análogos da ureia e de aminas secundárias, sendo a difenilamina (DPA), a etil centralite (EC), a akardite II (AK-II) e a 2-nitrodifenilamina (2-NDPA) os mais comuns. Alternativas menos tóxicas a esses compostos, já foram apontadas na literatura, como os ésteres e éteres aromáticos propostos por Krumlinde ${ }^{6}$ ou os estabilizantes à base de óleo epoxidado, conhecidos como Lankroflex E2307 (óleo de soja epoxidado), Lankroflex L (óleo de linhaça epoxidado), e Lankroflex ED6 (uma mistura epoxidada de ácidos graxos $\mathrm{C}_{14}-\mathrm{C}_{22}$ e 2-etil-hexil ésteres). ${ }^{1,7}$ Recentemente, surgiram também possibilidades de substituição dos estabilizantes por compostos naturais, chamados de "green stabilizers", sendo a $\alpha$-ionona apontada como o "green stabilizer" mais promissor

*e-mail: tanosfranca@gmail.com

"e-mail alternativo: letivan.br@gmail.com dentre outros compostos de origem natural como a Vitamina E e a Curcumina. ${ }^{8}$ Embora algumas das alternativas acima tenham se mostrado promissoras, ainda não há registros de um estabilizante comercial que não seja um análogo da ureia e de aminas secundárias.

\section{Revisão bibliográfica e estado da arte}

As N-nitroso-aminas e as N-nitroso-amidas oriundas da interação entre os estabilizantes comerciais e os vapores nitrosos gerados na degradação da NC, estão associadas ao aparecimento de carcinomas em diversos órgãos, variando o órgão afetado com o composto administrado. Identificou-se, por exemplo, a ocorrência de tumores de fígado em ratos pela ação da N-nitroso-dimetilamina (DMN). Já a N-nitroso-metil-ureia, foi capaz de provocar lesões hemorrágicas seguidas de carcinomas no estômago, intestino e pâncreas de cobaias. ${ }^{9,10}$

A Figura 1 ilustra o processo de decomposição de N-nitrosoaminas primárias formadas na degradação da NC. Iniciando por mecanismos do tipo $\mathrm{S}_{\mathrm{N}} 1$ ou $\mathrm{S}_{\mathrm{N}} 2$, as espécies resultantes sofrem rearranjo para diazo-hidróxidos. Em seguida, sofrem eliminação de água, gerando cátions diazônio $\mathrm{R}-\mathrm{N}_{2}{ }^{+}$, que após perderem o $\mathrm{N}_{2}$, geram carbocátions $\left(\mathrm{R}^{+}\right)$. Estes últimos, por serem eletrófilos, são capazes de alquilar nucleófilos do DNA (nas posições $\mathrm{N}^{7}$ e $\mathrm{O}^{6}$ da guanina e $\mathrm{N}^{1} \mathrm{da}$ adenina) ou formar diazoalcanos, catalisando alterações de sítios específicos da dupla hélice do DNA, que podem provocar mutações. ${ }^{11,12}$

As alquil-aminas secundárias ou as aminas aromáticas secundárias, como a difenilamina (DPA), geram N-nitroso-aminas com potencial de serem $\mathrm{CMR}^{14}$ (vide Figuras $1 \mathrm{~S}$ e $2 \mathrm{~S}$ do material suplementar). Todavia estas não se decompõem espontaneamente como as $\mathrm{N}$-nitroso-aminas derivadas de aminas primárias. A reação cessa com a formação de N-nitroso-amina (Figura 2), porque estas substâncias são bases muito fracas e insolúveis em ácidos diluídos.

As alquil-aminas terciárias não apresentam hidrogênio ligado ao átomo de nitrogênio. Possivelmente, o átomo de hidrogênio ligado ao átomo de carbono adjacente ao nitrogênio é eliminado para formação de produtos complexos. ${ }^{13}$ Já as aril-aminas terciárias sofrem a nitrosação do anel aromático via uma reação de substituição eletrofílica, conforme ilustrado na Figura 3.

As N-alquil-nitrosaminas possuem um mecanismo mutagênico comum, iniciado por uma ativação metabólica pelas enzimas do 
$1^{\text {a }}$ etapa: Rearranjo para um diazo hidróxido

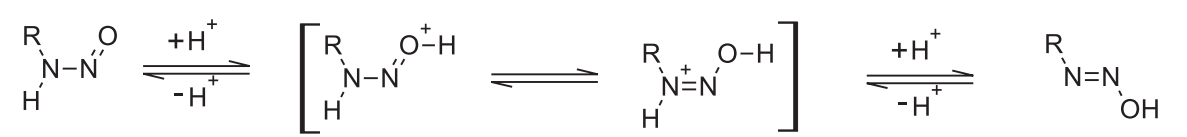

$2^{a}$ etapa: Perda de água para formar o cátion diazônio

Diazo hidróxido

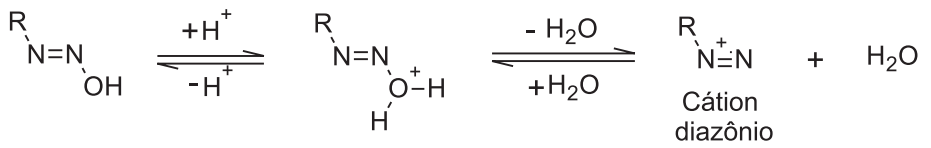

$3^{a}$ etapa: Perda de nitrogênio para formar um carbocátion

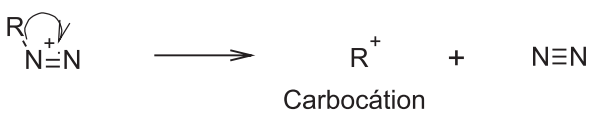

Figura 1. Mecanismo de decomposição das $N$-nitroso-aminas ${ }^{13}$ ( $R=$ radical alquil ou aril)

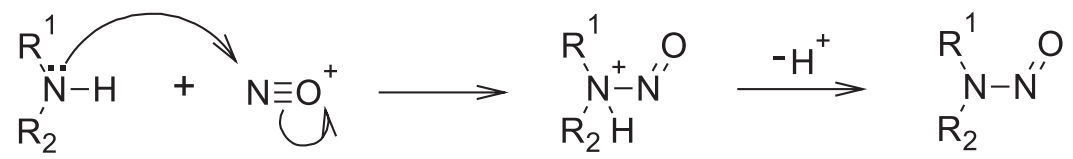

Figura 2. Mecanismo da nitrosação de aminas secundárias ${ }^{13}\left(R_{1}\right.$ e $R_{2}=$ radical alquil ou aril)

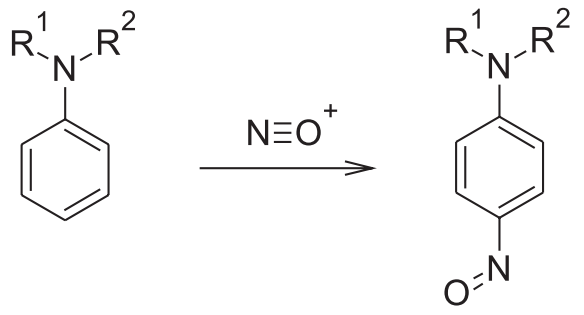

Figura 3. Nitrosação de amina terciária aromática ${ }^{13}$

citocromo P450, que tornam esses compostos mais polares e hidrossolúveis, através da hidroxilação do carbono adjacente ao grupo $\mathrm{N}$-nitroso (Figura 4). As únicas N-nitroso aminas que não passam por essa rota metabólica são aquelas que não possuem hidrogênio em carbono $\alpha$, tais como a $\mathrm{N}$-nitroso-metil-tercbutilamina, a N-nitrosoetil-tercbutilamina e a $\mathrm{N}$-nitroso-dibenzilamina. Possivelmente, por essa razão, elas não são carcinogênicas. ${ }^{11}$

As N-nitroso-amidas, que são os produtos da degradação da EC e da AK-II (vide Figuras S3 e S4 do material suplementar), se hidrolisam de maneira similar ao que ocorre na decomposição das N-nitroso-hidroxiaminas (Figura 4) e nessa decomposição também são formados diazo-alcanos capazes de interagir com o DNA (Figura 5).

Os danos provocados nas bases de DNA pelas N-nitroso-aminas e N-nitroso-amidas podem levar a lesões citotóxicas e mutagênicas. ${ }^{12}$ As lesões citotóxicas bloqueiam a replicação levando à morte celular, enquanto as lesões mutagênicas provocam erros de codificação e causam mutações no DNA, que são consideradas como um mecanismo importante de carcinogênese.

Comparando as estruturas e atividades cancerígenas conhecidas de vários compostos $\mathrm{N}$-nitrosados, pode-se afirmar que as $\mathrm{N}$-nitroso dialquil aminas produzem tumores em órgãos distantes de sua introdução, onde provavelmente existe a capacidade de hidroxilar o carbono do composto nitrosado. Já as N-nitroso amidas apenas induzem a formação de tumores no local do seu contato, provavelmente, devido à menor solubilidade em meio aquoso, o que faz com que estes compostos não sejam transportados para longe do local de sua aplicação. Consequentemente, os diazoalcanos responsáveis pelo câncer serão<smiles>[R2]CN([R])N=O</smiles>

\section{N-Nitroso amina}

$\mathrm{N}-$ Nitroso hidroxiamina

Figura 4. Metabolização de N-nitroso-aminas ${ }^{15}$<smiles>[R]N(N=O)C(C)=O</smiles>

N-Nitroso amida<smiles>CC(=O)O</smiles><smiles>[R]N=NO</smiles>

Alquil diazo hidróxido 
produzidos no local do contato com a pele. Isso acontece também no caso de estabilizantes da família da ureia, como a AK-II e a EC. ${ }^{11}$

$\mathrm{O}$ desenvolvimento da mutagenicidade em bactérias foi investigado a partir de cinco $\mathrm{N}$-nitrosaminas ${ }^{15}$ - N-nitroso-dimetilamina (NDMA), N-nitroso-dietilamina (NDEA), N-nitroso-di-n-propilamina (NDPrA), N-nitrosopirrolidina (NPYR) e N-nitroso-difenilamina (NNDPA) - durante processos oxidativos envolvendo fotólise UV, ozônio e radicais $\mathrm{OH}$. Durante a fotólise UV, a mutagenicidade foi detectada apenas no caso da NNDPA. Os produtos de oxidação de NDMA, NDEA e NDPrA não apresentaram mutagenicidade significativa nas cepas utilizadas. Em contrapartida, as oxidações de NNDPA e NPYR por radicais hidroxílicos indicaram a formação de espécies mutagênicas. Dessa maneira, a associação entre a contaminação causada pela manipulação direta da DPA e a exposição ao sol aumenta os riscos de ocorrência de cânceres de pele. ${ }^{12}$

A literatura registra também que o potencial cancerígeno depende das cadeias laterais e aumenta da seguinte forma para as $\mathrm{N}$-nitroso aminas: $\mathrm{N}, \mathrm{N}$-diaril-N-NO $<\mathrm{N}$-aril-N-NO $<\mathrm{N}, \mathrm{N}$-dialquil-N-NO. ${ }^{16}$ Portanto, as $\mathrm{N}$-nitroso-alquil-anilinas, produzidas a partir da EC, seriam mais tóxicas que as N-nitroso-DPAs, derivadas da DPA ou da 2-NDPA.

De forma geral, o uso dos estabilizantes EC, AK-II, DPA e 2-NDPA em quase todas as composições de propelentes em todo o mundo faz com que esses materiais sejam considerados tóxicos, muito tóxicos, cancerígenos ou mutagênicos. No entanto, para uma informação mais precisa e quantitativa a respeito dos riscos associados a cada um deles, é necessário avaliar seus potenciais CMR, bem como os de seus produtos de degradação. A investigação conduzida no presente trabalho é inédita na literatura e permite estabelecer uma hierarquia de riscos desses estabilizantes. Com essa informação, equipes de segurança do trabalho e de avaliação de risco ambiental podem ter dados mais confiáveis referentes à fabricação, à manipulação e às operações de descarte de propelentes.

\section{PARTE EXPERIMENTAL}

Os principais produtos de degradação dos quatro estabilizantes investigados no presente trabalho - DPA, EC, AK-II, 2-NDPA - e que são os mais utilizados em propelentes à base de NC, estão listados na Tabela 1. A partir das estruturas destes compostos é possível prever seus riscos toxicológicos utilizando o software Lazar (Lazy Structure-activity Relationships) disponível em https://lazarin-silico. de/predict. ${ }^{17,18}$ Este software gera previsões para uma variedade de propriedades tóxicas com base em compostos reportados com estruturas semelhantes, sendo estruturado na OpenTox, ${ }^{19}$ a principal

Tabela 1. Principais produtos de degradação dos estabilizantes de propelentes

\begin{tabular}{llc}
\hline Estabilizante & Principais produtos de degradação* & Referências \\
\hline DPA & N-nitroso-difenilamina (NNDPA) & 16,20 \\
& 2-NDPA & \\
& 2-nitro-difenilamina (4-NDPA) & \\
\hline 2-NDPA & 2-N-nitroso-difenilamina (2-NNDPA) & 21,22 \\
& 2,4-dinitro-difenilamina (2,4'-DNDPA) & \\
\hline EC & N-nitroso-etilanilina (NNEA) & 23 \\
& 4-nitro-N-nitroso-etilanilina (4-NNNEA) & \\
& 4-nitro-etil-centralite (4-NEC) & \\
& 4,4'-dinitro-etil-centralite (4,4'-DNEC) & \\
\hline AK-II & N-nitroso-akardite-II (NNAK-II) \\
& 2-nitro-akardite-II (2-NAK-II) \\
& 4-nitro-akardite-II (4-NAK-II) \\
& N-nitroso-2-nitro-akardite-II (2-NNNAK-II) \\
\hline
\end{tabular}

*As estruturas dos produtos de degradação são apresentadas nas Figuras 1S a $4 \mathrm{~S}$ do material suplementar. plataforma global aberta para toxicologia preditiva, disponível em http://www.opentox.net. O usuário tem a opção de desenhar ou inserir a representação química com os caracteres Smiles (Simplified Molecular Input Line Entry Specification), que será a entrada de dados para a predição no Lazar. ${ }^{17,18}$

O software Lazar ${ }^{17,18}$ fornece seus resultados com base nas opções de escolha do usuário, que seriam os valores preditivos da toxicidade aguda para o peixe Fathead Minnow ${ }^{25}$ e para o crustáceo planctônico Daphnia Magna, ${ }^{26}$ que são organismos bioindicadores, bem como a estimativa de permeabilidade à barreira hematoencefálica ${ }^{27}(\mathrm{BHE}) \mathrm{e}$ os valores de toxicidade frente à bactéria Salmonela Typhimurium. ${ }^{28}$ Além disso, o Lazar ${ }^{17,18}$ também apresenta nos resultados da predição, os compostos similares utilizados como referência de suas aproximações, sendo confiável se a estrutura investigada estiver dentro do domínio de aplicabilidade do OpenTox. ${ }^{17,18}$

Os cálculos associados à toxidade correspondem às probabilidades de ocorrência do risco toxicológico, sendo positivos para penetração na BHE, carcinogenicidade e mutagenicidade ou negativos para não penetração na BHE, não carcinogenicidade e não mutagenicidade. Ao invés de se trabalhar com nível de confiança, os resultados podem ser interpretados como probabilidades reais variando de 0 a 1. Uma previsão confiável tem uma alta probabilidade para a classe prevista e uma baixa probabilidade para a outra. As previsões não confiáveis, ou seja, não conclusivas possuem valores semelhantes para ambas às classes e são causadas por muitas atividades contraditórias de compostos similares. As similaridades são calculadas a partir do método de Tanimoto-Jaccard..$^{29,30}$

O Lazar ${ }^{17,18}$ faz previsões de toxicologia baseadas em algoritmos estatísticos através da análise de fragmentos estruturais em um conjunto de moléculas com estruturas similares, capturando apenas os fragmentos relevantes para o end point ou limite tóxico sob investigação. ${ }^{31}$ Um estudo comparativo com outros aplicativos computacionais revelou que o Lazar apresentou as piores estatísticas entre os modelos para carcinogenicidade em hamsters, entretanto, foi o mais preciso para a predição da mutagenicidade. ${ }^{32}$

\section{RESULTADOS E DISCUSSÃO}

\section{Validação da metodologia}

Para validar a metodologia utilizada foi feita uma pesquisa na literatura de alguns resultados já reportados e, no caso de valores numéricos, como a $\mathrm{DL}_{50}$, os resultados pesquisados foram comparados com os fornecidos pelo Lazar ${ }^{17,18}$ (Tabela 2). Os resultados qualitativos, como a carcinogenicidade e a mutagenicidade, também foram comparados com os resultados pesquisados na literatura. Todos os compostos analisados nesta validação tem boa penetração na BHE porque são lipossolúveis. ${ }^{33} \mathrm{~A} \mathrm{DL}_{50}$ não foi incluída na Tabela 2 porque os resultados na literatura também serão valores estimados com outros modelos preditivos. Pode-se afirmar que a Tabela 2 mostra uma congruência da maioria dos dados preditos com a literatura, especialmente, para a predição da mutagenicidade e carcinogenicidade.

Ainda sobre a validação de resultados foi reportada uma pesquisa envolvendo predições de mutagenicidade com os dados de 3.895 estruturas e seus resultados foram confirmados com a utilização deste aplicativo computacional. Com base nesse trabalho a mutagenicidade com a Salmonella poderia ser prevista com precisão de $85 \%$ para compostos no domínio do banco de dados das substâncias com potencial carcinogênico. A precisão para outras carcinogenicidades variaram entre 78 e $95 \%$ para estruturas dentro do domínio de aplicabilidade. ${ }^{17}$ Em outro reporte similar foram utilizados 1.544 compostos químicos potencialmente carcinogênicos a ratos e o aplicativo computacional atingiu $78 \%$ de especificidade..$^{34}$ 
Em outro exemplo, o uso deste aplicativo computacional foi bem sucedido em três casos ligados a produtos químicos relacionados à reciclagem em um aterro industrial da Itália. O primeiro caso é uma avaliação de ensaios in vitro para a investigação da toxicidade de um lixiviado. O segundo exemplo analisa qualitativamente o potencial carcinogênico de alguns compostos perfluorados, usando modelos $Q S A R$ e células in vitro. Finalmente, uma avaliação QSAR de diferentes produtos químicos foi demonstrada, a fim de se validar como modelos in silico seriam utilizados, como ferramenta, para preencher as lacunas existentes na literatura, sobre o perfil toxicológico dos compostos investigados. ${ }^{35}$

Em síntese, a falta de dados de algumas substâncias deste trabalho evidencia a escassez de material publicado sobre o assunto e fortalece a necessidade de mais estudos sobre a toxicidade de materiais energéticos para se preencher esta lacuna na literatura científica, com também, o uso de aplicativos computacionais de predição toxicológica. Foram encontradas algumas referências que já utilizam dados de toxicologia preditiva. ${ }^{36,37}$

\section{Análise de toxicidade dos estabilizantes}

Os resultados da análise de toxicidade dos estabilizantes DPA, EC, AK-II e 2-NDPA, através do Lazar, ${ }^{17,18}$ são apresentados na Tabela 3 expressos na forma de probabilidades de ocorrência do risco e de dados adicionais, como, por exemplo, a dose letal para ratos e camundongos. Os dados apresentados permitem inferir que todos os estabilizantes tendem a ter uma boa penetração na BHE, o que implica em uma boa difusão pelo corpo humano. A DPA é, provavelmente, o estabilizante mais tóxico seguido da 2-NDPA, que é um de seus derivados. Ambos apresentaram potencial mutagênico. Entre a EC e a AK-II, pode-se inferir que, possivelmente, não são carcinogênicos nem mutagênicos.

A Tabela 4 apresenta as moléculas utilizadas pelo $\operatorname{Lazar}^{17,18}$ para determinar os valores de probabilidade propostos na Tabela 3 , de acordo com as atividades tóxicas conhecidas, indicando a sua similaridade com a estrutura do estabilizante investigado. Adicionalmente, a Tabela 5 apresenta os resultados similares para os principais produtos de degradação de cada estabilizante investigado.

A análise da Tabela 5 mostra que os produtos derivados da degradação da DPA apresentaram potencial de serem tóxicos e mutagênicos, podendo ser hierarquizados quanto à mutagenicidade na seguinte ordem: 4-NDPA > 2-NDPA > NNDPA e quanto à toxicidade na seguinte ordem: NNDPA > 4-NDPA > 2-NDPA, todos com boa penetração na BHE. Já os produtos derivados da degradação da 2-NDPA apresentaram grande potencial mutagênico na seguinte ordem: 2,4'-DNDPA > 4-amino-bifenil > 2-NNDPA, e alta toxicidade na ordem inversa.

Os produtos derivados da degradação da EC apresentaram os melhores resultados para penetração na BHE em comparação com os demais estabilizantes. O potencial mutagênico desses produtos foi hierarquizado na seguinte ordem: 4-NNEA 4-NEC > 4,4'-DNEC 2,4'-DNEC 2,4-DNEC > NEA. Foram também preditas as altíssimas toxicidades da 4-NNNEA e da 4-NEA respectivamente.

Os produtos derivados da degradação da AK-II e da NNAK-II apresentaram potencial carcinogênico e alta toxicidade quando comparados aos demais produtos desta família. O potencial mutagênico observado para esses compostos segue a seguinte ordem: 4-NAK-II > 2-NAK-II > 2-NNAK-II > NNAK-II.

Tabela 2. Validação da metodologia utilizada

\begin{tabular}{|c|c|c|c|c|c|c|c|c|c|}
\hline Composto & & $\begin{array}{c}\text { Toxicidade } \\
\text { aguda (Fathead } \\
\text { Minnow em } \\
\left.\mathrm{mg} \mathrm{L}^{-1}\right)\end{array}$ & $\begin{array}{c}\text { Toxicidade } \\
\text { aguda (Daphnia } \\
\text { Magna em } \\
\left.\mathrm{mg} \mathrm{L}^{-1}\right)\end{array}$ & $\begin{array}{c}\text { Penetração } \\
\text { BHE }\end{array}$ & $\begin{array}{l}\text { Carcinogenici- } \\
\text { dade (camun- } \\
\text { dongos) }\end{array}$ & $\begin{array}{c}\mathrm{DL}_{50} \text { Camun- } \\
\text { dongos } \\
(\mathrm{mg} / \mathrm{kg} / \mathrm{dia})\end{array}$ & $\begin{array}{l}\text { Carcinogenici- } \\
\text { dade (Ratos) }\end{array}$ & $\begin{array}{l}\mathrm{DL}_{50} \text { Ratos } \\
(\mathrm{mg} / \mathrm{kg} / \mathrm{dia})\end{array}$ & $\begin{array}{l}\text { Mutagenicida- } \\
\text { de (Salmonella } \\
\text { typhimurium) }\end{array}$ \\
\hline \multirow{2}{*}{ Benzeno } & Lazar & 7,560 & 5,86 & Penetra & Não C & 0,0627 & $\mathrm{C}$ & 0,037 & M \\
\hline & Literatura & $15,100^{38}$ & $2,00^{39}$ & Penetra & $\mathrm{C}^{40}$ & $2,195^{41}$ & $\mathrm{C}^{40}$ & $0,0137^{41}$ & $\mathbf{M}^{42}$ \\
\hline \multirow{2}{*}{ Naftaleno } & Lazar & 3,35 & 0,39 & Penetra & Não C & 0,002 & $\mathrm{C}$ & 0,0105 & M \\
\hline & Literatura & $7,900^{38}$ & $8,6^{43}$ & Penetra & Não C ${ }^{44}$ & $5,100^{41}$ & $\mathrm{C}^{45}$ & $2,600^{41}$ & $\mathbf{M}^{46}$ \\
\hline \multirow{2}{*}{ Tolueno } & Lazar & 9,13 & 91,7 & Penetra & Não C & 0,109 & Não C & 3,06 & M \\
\hline & Literatura & $9,39^{47}$ & $310^{43}$ & Penetra & Não $C^{40}$ & $2,15^{48}$ & Não $C^{40}$ & $5,50^{48}$ & $\mathbf{M}^{49}$ \\
\hline \multirow{2}{*}{ Fenol } & Lazar & 3,91 & 9,58 & Penetra & Não C & 0,326 & $\mathrm{C}$ & 0,137 & Não M \\
\hline & Literatura & $12^{43}$ & $12^{43}$ & Penetra & Não $C^{50}$ & $0,340^{51}$ & Não $C^{50}$ & $0,300^{41}$ & Não $M^{46}$ \\
\hline
\end{tabular}

Tabela 3. Predição da periculosidade dos estabilizantes clássicos pelo Lazar $^{17,18}$

\begin{tabular}{|c|c|c|c|c|c|c|c|c|c|c|}
\hline Estabilizante & $\begin{array}{l}\text { Toxicidade } \\
\text { aguda } \\
\text { (Fathead } \\
\text { Minnow em } \\
\mathrm{mg} \mathrm{L}^{-1} \text { ) }\end{array}$ & $\begin{array}{l}\text { Toxicidade } \\
\text { aguda } \\
\text { (Daphnia } \\
\text { Magna em } \\
\mathrm{mg} \mathrm{L}^{-1} \text { ) }\end{array}$ & $\begin{array}{c}\text { Penetração } \\
\text { BHE }\end{array}$ & $\begin{array}{l}\text { Carcino- } \\
\text { genicidade } \\
\text { (múltiplos } \\
\text { roedores) }\end{array}$ & $\begin{array}{l}\text { Carcinoge- } \\
\text { nicidade (ca- } \\
\text { mundongos) }\end{array}$ & $\begin{array}{c}\mathrm{DL}_{50} \\
\text { Camundon- } \\
\text { gos }\left(\mathrm{mg} \mathrm{kg}^{-1}\right)\end{array}$ & $\begin{array}{c}\text { Carcino- } \\
\text { genicidade } \\
\text { (Ratos) }\end{array}$ & $\begin{array}{c}\mathrm{DL}_{50} \text { Ratos } \\
\left(\mathrm{mg} \mathrm{kg}^{-1}\right)\end{array}$ & $\begin{array}{c}\text { Dose diária } \\
\text { máxima a } \\
\text { humanos } \\
\left(\mathrm{mg} \mathrm{kg}^{-1}\right)\end{array}$ & $\begin{array}{c}\text { Mutage- } \\
\text { nicidade } \\
\text { (Salmonella } \\
\text { typhimurium) }\end{array}$ \\
\hline$D P A$ & 80,8 & 6,45 & $\begin{array}{l}+: \mathbf{0 , 2 2 2} \\
-: 0,0284\end{array}$ & $\begin{array}{l}+: \mathbf{0 , 5 1 7} \\
-: 0,483\end{array}$ & $\begin{array}{l}+: 0,449 \\
-\mathbf{:} \mathbf{0 , 5 5 1}\end{array}$ & 0.195 & $\begin{array}{l}+: \mathbf{0 , 5 6 1} \\
-: 0,439\end{array}$ & 0,0635 & 6,25 & $\begin{array}{c}+: \mathbf{0 , 6 6} \\
-: 0,34\end{array}$ \\
\hline $2-N D P A$ & 23,0 & 8,25 & $\begin{array}{c}+\mathbf{0 , 1 6 5} \\
-: 0,0204\end{array}$ & $\begin{array}{l}+: \mathbf{0 , 2 6 3} \\
-: 0,237\end{array}$ & $\begin{array}{l}+: 0,234 \\
-\mathbf{0} \mathbf{0 , 2 6 6}\end{array}$ & 0,311 & $\begin{array}{l}+: \mathbf{0 , 2 5 4} \\
-: 0,246\end{array}$ & 0,207 & -- & $\begin{array}{l}+: \mathbf{0 , 5 7 7} \\
-: 0,123\end{array}$ \\
\hline$E C$ & -- & 71,8 & $\begin{array}{l}+: \mathbf{0 , 2} \\
-: 0,0\end{array}$ & $\begin{array}{l}+: \mathbf{0 , 0 9 5 1} \\
-: 0,0715\end{array}$ & $\begin{array}{l}+: 0,0737 \\
\text { - : } \mathbf{0 , 0 9 2 9}\end{array}$ & -- & $\begin{array}{l}+: 0,0825 \\
-: \mathbf{0 , 0 8 4 2}\end{array}$ & -- & -- & $\begin{array}{l}+: 0,134 \\
-\mathbf{0} \mathbf{0 , 1 3 9}\end{array}$ \\
\hline$A K-I I$ & -- & -- & $\begin{array}{c}+: \mathbf{0 , 2 8 6} \\
-: 0,0\end{array}$ & $\begin{array}{c}+: \mathbf{0 , 1 0 1} \\
-: 0,0986\end{array}$ & $\begin{array}{l}+: 0,085 \\
-: \mathbf{0 , 1 1 5}\end{array}$ & -- & $\begin{array}{l}+: 0,0752 \\
-: \mathbf{0 , 0 9 1 5}\end{array}$ & -- & -- & $\begin{array}{c}+: 0,24 \\
-\mathbf{0} \mathbf{0 , 2 6}\end{array}$ \\
\hline
\end{tabular}


Tabela 4. Moléculas com maior similaridade utilizadas pelo banco de dados no Lazar ${ }^{17,18}$ para previsão da periculosidade dos estabilizantes clássicos

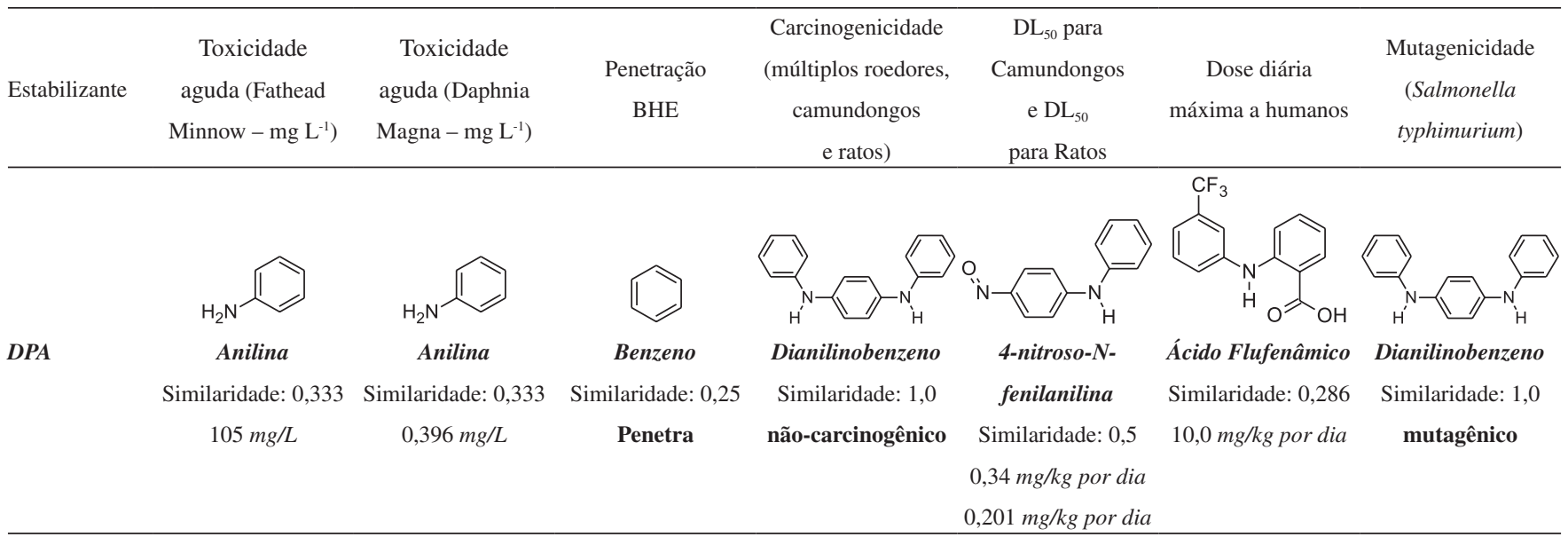

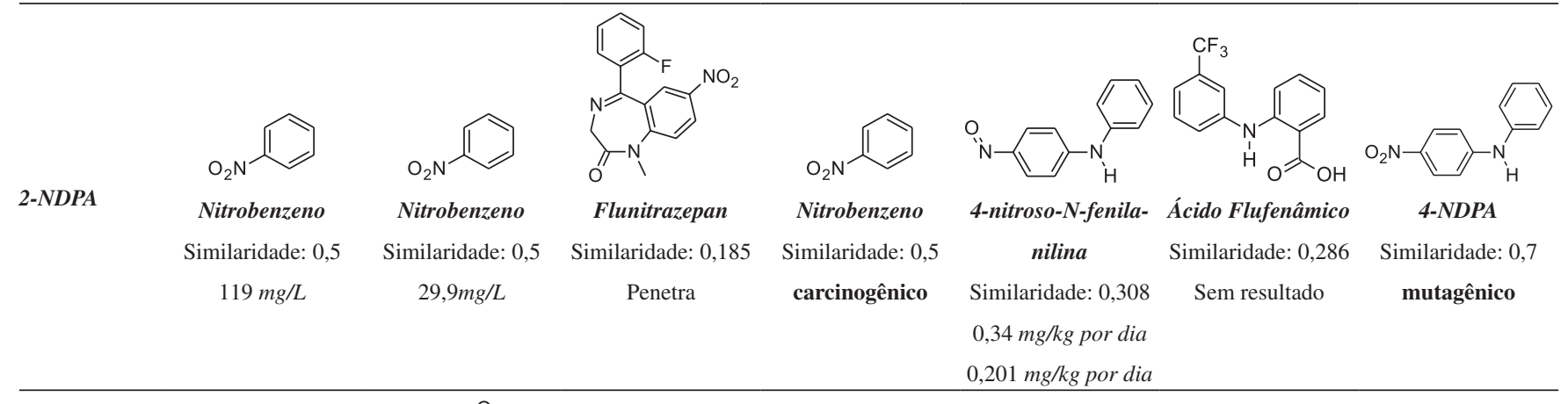

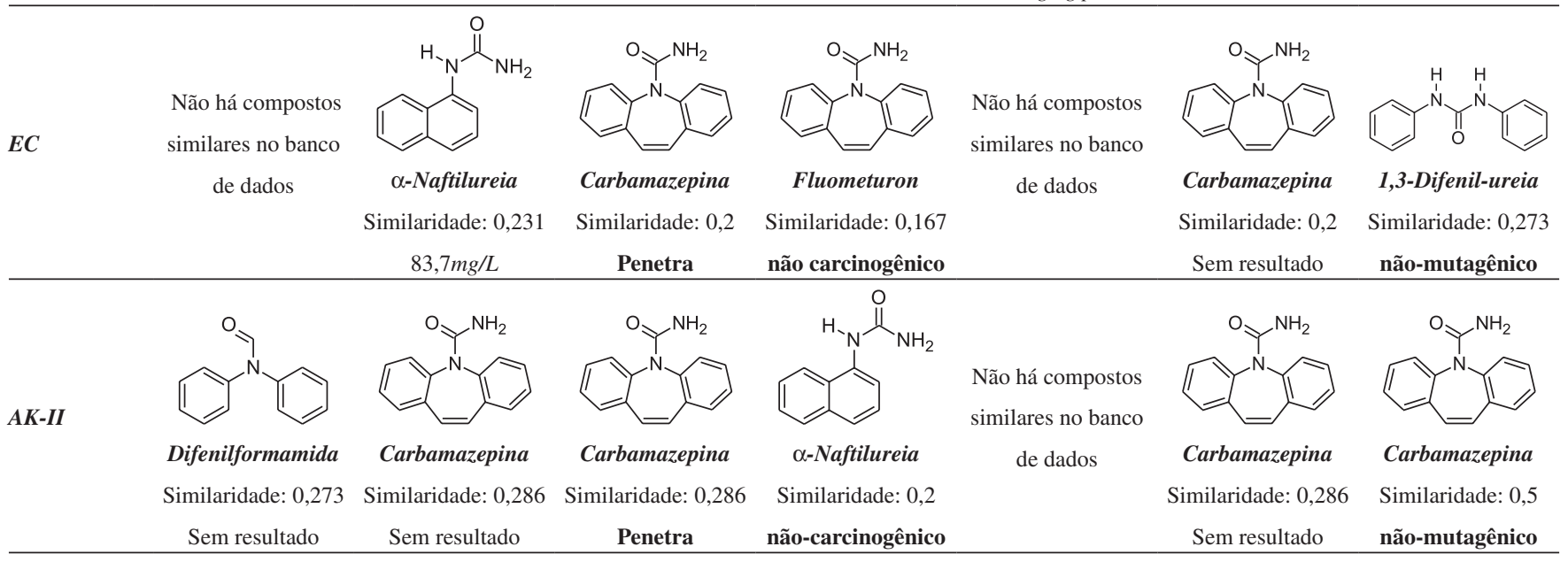

\section{CONCLUSÃO}

Com base nos resultados obtidos, pode-se afirmar que, dentre os quatro estabilizantes investigados a DPA e a 2-NDPA apresentam grande potencial mutagênico, sendo a DPA a mais tóxica seguida pela 2-NDPA. Além disso, os quatro estabilizantes investigados apresentaram índices que sugerem grande capacidade de penetração na BHE, possuindo desta forma capacidade de transporte e de difusão pela corrente sanguínea no organismo humano.

Com base na diferença entre as probabilidades encontradas, os produtos de degradação dos quatro estabilizantes clássicos, foram hierarquizados quanto ao potencial mutagênico, estabelecendo a seguinte ordem: 2,4'-DNDPA > 4-amino-bifenil $>4$-NDPA $>$ 2-NDPA > 4-NEC 4-NNEA 4-NAK-II. Como consequência desse resultado, os quatro estabilizantes foram ordenados quanto ao potencial mutagênico de seus produtos de degradação em: 2-NDPA $>$ DPA $>$ EC $>$ AK-II.
Com relação ao potencial carcinogênico, todos os produtos apresentaram probabilidades próximas impossibilitando uma análise comparativa confiável. A única exceção foi a NNAK-II, derivada da AK-II, que apresentou este risco em potencial.

Sobre a toxicidade, com base nas probabilidades da $\mathrm{DL}_{50}$ em ratos e camundongos, foi possível propor a seguinte ordem decrescente de toxicidade para os produtos de degradação: 4 -NNNEA $>4$-NEA $~$ NNAK-II $>2$-NNDPA $\sim$ NNDPA > 4-amino-bifenil $>4$-NDPA $>2$-NDPA $>2,4$ '-NDPA e, consequentemente, a seguinte ordem de toxicidade: $\mathrm{EC}>\mathrm{AK}-\mathrm{II}>2$-NDPA $>$ DPA para os estabilizantes clássicos com relação aos seus produtos da degradação.

Por fim, foi possível observar que o maior potencial mutagênico está associado ao produto da degradação que contêm grupos nitro em sua estrutura, enquanto que a toxicidade está relacionada à presença do grupo nitroso. 
Tabela 5. Predição da toxicidade dos produtos de degradação dos estabilizantes clássicos pelo Lazar ${ }^{17,18}$

\begin{tabular}{|c|c|c|c|c|c|c|c|c|c|c|c|}
\hline Estabilizante & $\begin{array}{c}\text { Produtos } \\
\text { Derivados }\end{array}$ & $\begin{array}{l}\text { Toxicida- } \\
\text { de aguda } \\
\text { (Fathead } \\
\text { Minnow - } \\
\text { mg/L) }\end{array}$ & $\begin{array}{c}\text { Toxicida- } \\
\text { de aguda } \\
\text { (Daphnia } \\
\text { Magna - } \\
\text { mg/L) }\end{array}$ & $\begin{array}{c}\text { Penetração } \\
\text { BHE }\end{array}$ & $\begin{array}{c}\text { Carcino- } \\
\text { genicidade } \\
\text { (múltiplos } \\
\text { roedores) }\end{array}$ & $\begin{array}{c}\text { Carcino- } \\
\text { genicidade } \\
\text { (camundon- } \\
\text { gos) }\end{array}$ & $\begin{array}{l}\mathrm{DL}_{50} \mathrm{Ca}- \\
\text { mundongos } \\
(\mathrm{mg} / \mathrm{kg})\end{array}$ & $\begin{array}{l}\text { Carcino- } \\
\text { genicidade } \\
\text { (Ratos) }\end{array}$ & $\begin{array}{c}\mathrm{DL}_{50} \text { Ratos } \\
(\mathrm{mg} / \mathrm{kg})\end{array}$ & $\begin{array}{c}\text { Dose diária } \\
\text { máxima a } \\
\text { humanos } \\
(\mathrm{mg} / \mathrm{kg})\end{array}$ & $\begin{array}{c}\text { Mutage- } \\
\text { nicidade } \\
\text { (Salmonella } \\
\text { typhi- } \\
\text { murium) }\end{array}$ \\
\hline \multirow{3}{*}{$D P A$} & $2-N D P A$ & 23 & 8,25 & $\begin{array}{c}+: \mathbf{0 , 1 6 5} \\
-: 0,0204\end{array}$ & $\begin{array}{l}+: \mathbf{0 , 2 6 3} \\
-: 0,237\end{array}$ & $\begin{array}{l}+: 0,234 \\
-: \mathbf{0 , 2 6 6}\end{array}$ & 0.311 & $\begin{array}{l}+: \mathbf{0 , 2 5 4} \\
-: 0,246\end{array}$ & 0,207 & \multirow{3}{*}{22,7} & $\begin{array}{l}\text { +: } \mathbf{0 , 5 7 7} \\
\text { - : } 0,123\end{array}$ \\
\hline & 4-NDPA & 16,6 & 42,3 & $\begin{array}{c}+: \mathbf{0 , 2 1 5} \\
-: 0,0253\end{array}$ & $\begin{array}{l}+: \mathbf{0 , 3 8 7} \\
-: 0,363\end{array}$ & $\begin{array}{l}+: 0,344 \\
-: \mathbf{0 , 4 0 6}\end{array}$ & 0.258 & $\begin{array}{l}+: \mathbf{0 , 3 7 8} \\
-: 0,372\end{array}$ & 0,162 & & $\begin{array}{l}+\mathbf{0} \mathbf{0 , 6 2} \\
-: 0,13\end{array}$ \\
\hline & NNDPA & 163 & 2,99 & $\begin{array}{c}+: \mathbf{0 , 1 4 9} \\
-: 0,0177\end{array}$ & $\begin{array}{l}+: \mathbf{0 , 1 8 8} \\
-: 0,112 \\
\end{array}$ & $\begin{array}{l}+: \mathbf{0 , 1 3 5} \\
-: 0,115\end{array}$ & 0.203 & $\begin{array}{l}+: \mathbf{0 , 1 9} \\
-: 0,11 \\
\end{array}$ & 0,0339 & & $\begin{array}{c}+\mathbf{0} \mathbf{0 , 2 1 1} \\
-: 0,0893\end{array}$ \\
\hline \multirow{3}{*}{$2-N D P A$} & 2-NNDPA & 26,3 & 1,85 & $\begin{array}{l}+: \mathbf{0 , 1 3 7} \\
-: 0,035\end{array}$ & $\begin{array}{l}+\mathbf{0} \mathbf{0 , 3 0 6} \\
-: 0,239\end{array}$ & $\begin{array}{c}+: 0,256 \\
-: \mathbf{0 , 2 9}\end{array}$ & & $\begin{array}{l}+\mathbf{0} \mathbf{0 , 2 8 7} \\
-: 0,259\end{array}$ & 0,034 & & $\begin{array}{c}\text { +: } \mathbf{0 , 4 5 5} \\
-: 0,0909\end{array}$ \\
\hline & $2,4^{\prime}-D N D P A$ & 14,7 & 16,8 & $\begin{array}{l}+: \mathbf{0 , 1 9 5} \\
-: 0,0277\end{array}$ & $\begin{array}{l}+: 0,299 \\
-: \mathbf{0 , 3 0 1}\end{array}$ & $\begin{array}{l}+: 0,271 \\
-: \mathbf{0 , 3 2 9}\end{array}$ & 0,397 & $\begin{array}{l}+: 0,289 \\
-: \mathbf{0 , 3 1 1}\end{array}$ & 0,238 & 27,8 & $\begin{array}{l}+: \mathbf{0 , 7 4 4} \\
-: 0,156\end{array}$ \\
\hline & $\begin{array}{c}\text { 4-amino- } \\
\text { bifenil }\end{array}$ & 25,7 & 3,43 & $\begin{array}{c}+: \mathbf{0 , 2 1 2} \\
-: \mathbf{0 , 0 8 1 7}\end{array}$ & $\begin{array}{l}+: \mathbf{0 , 5 1 3} \\
-: 0,345\end{array}$ & $\begin{array}{l}+: \mathbf{0 , 4 7 8} \\
-: 0,379\end{array}$ & 0,033 & $\begin{array}{l}+: \mathbf{0 , 5 1 6} \\
-: 0,341\end{array}$ & 0,0454 & 5,9 & $\begin{array}{l}+: \mathbf{0 , 6 8 5} \\
-: 0,173\end{array}$ \\
\hline \multirow{6}{*}{$E C$} & NNEA & 25,5 & 1,7 & $\begin{array}{c}+: \mathbf{0 , 1 2 5} \\
-:: 0,0\end{array}$ & $\begin{array}{c}+: \mathbf{0 , 3 4 6} \\
-: 0,154\end{array}$ & $\begin{array}{l}+: \mathbf{0 , 1 4 9} \\
-: 0,123\end{array}$ & \multirow[t]{6}{*}{0,147} & $\begin{array}{l}+: \mathbf{0 , 3 4 7} \\
-: 0,153\end{array}$ & 0,00168 & & $\begin{array}{l}+: \mathbf{0 , 3 6 9} \\
-: 0,131\end{array}$ \\
\hline & 4-NNNEA & 18,4 & 21,7 & $\begin{aligned}+ & : \mathbf{0 , 1 7 2} \\
- & : 0,0\end{aligned}$ & $\begin{array}{l}+: \mathbf{0 , 2 1 5} \\
-: 0,202\end{array}$ & $\begin{array}{l}+: 0,178 \\
-: \mathbf{0 , 2 3 9}\end{array}$ & & $\begin{array}{l}+: 0,288 \\
-: \mathbf{0 , 3 2 8}\end{array}$ & \multirow[t]{5}{*}{0,000364} & & $\begin{array}{l}+: \mathbf{0 , 4 9 5} \\
-: 0,0882\end{array}$ \\
\hline & $4-N E C$ & 31,8 & 45,2 & $\begin{array}{c}+: \mathbf{0 , 2 4 1} \\
-:: 0,0\end{array}$ & $\begin{array}{l}+: \mathbf{0 , 2 4 1} \\
-: 0,221\end{array}$ & $\begin{array}{l}+: 0,215 \\
-: \mathbf{0 , 2 4 6}\end{array}$ & & $\begin{array}{c}+: 0,222 \\
-: \mathbf{0 , 2 4}\end{array}$ & & & $\begin{array}{l}\text { +: } \mathbf{0 , 5 1 1} \\
\text {-: } 0,104\end{array}$ \\
\hline & 4,4'-DNEC & 34,2 & 28,3 & $\begin{array}{c}+: \mathbf{0 , 2 0 7} \\
-:: 0,0\end{array}$ & $\begin{array}{l}+: 0,191 \\
-: \mathbf{0 , 1 9 4}\end{array}$ & $\begin{array}{l}+: 0,173 \\
-: \mathbf{0 , 2 1 2}\end{array}$ & & $\begin{array}{l}+: 0,174 \\
-: \mathbf{0 , 2 1 1}\end{array}$ & & & $\begin{array}{l}+: \mathbf{0 , 4 5 7} \\
-: 0,0815\end{array}$ \\
\hline & $2,4^{\prime}-D N E C$ & 38,5 & 52,7 & $\begin{array}{c}+: \mathbf{0 , 2 2 6} \\
-:: 0,0\end{array}$ & $\begin{array}{l}+: \mathbf{0 , 2 0 1} \\
-: \text { : } 0,199\end{array}$ & $\begin{array}{l}+: 0,181 \\
-: \mathbf{0 , 2 1 9}\end{array}$ & & $\begin{array}{l}+: 0,185 \\
-: \mathbf{0 , 2 1 5}\end{array}$ & & & $\begin{array}{l}\text { +: 0,447 } \\
-: 0,0866\end{array}$ \\
\hline & 2,4-DNEC & 22,5 & 55,5 & $\begin{array}{c}+: \mathbf{0 , 2 1 9} \\
-: 0,0\end{array}$ & $\begin{array}{l}+: 0,187 \\
-: \mathbf{0 , 1 8 8}\end{array}$ & $\begin{array}{l}+: 0,167 \\
-: \mathbf{0 , 2 0 8}\end{array}$ & & $\begin{array}{l}+: 0,171 \\
-: \mathbf{0 , 2 0 4}\end{array}$ & & & $\begin{array}{l}+: \mathbf{0 , 4 2 2} \\
-: 0,0783\end{array}$ \\
\hline \multirow{4}{*}{$A K-I I$} & NNAK-II & & & $\begin{array}{l}+: \mathbf{0 , 2 2 2} \\
-: 0,0284\end{array}$ & $\begin{array}{l}+: \mathbf{0 , 3 5 3} \\
-: 0,0641\end{array}$ & $\begin{array}{l}+: \mathbf{0 , 2 5 8} \\
-: 0,159\end{array}$ & & $\begin{array}{c}+: \mathbf{0 , 3 1 8} \\
-: 0,0985\end{array}$ & \multirow[t]{4}{*}{0,00168} & \multirow[t]{4}{*}{51,0} & $\begin{array}{l}+: \mathbf{0 , 2 5 4} \\
-: 0,163\end{array}$ \\
\hline & $2-N A K-I I$ & 30,6 & 15,1 & $\begin{array}{l}+: \mathbf{0 , 2} \\
-: 0,0\end{array}$ & $\begin{array}{l}+\mathbf{0} \mathbf{0 , 1 7 6} \\
-: 0,157\end{array}$ & $\begin{array}{l}+: 0,157 \\
-\mathbf{0 , 1 7 6}\end{array}$ & & $\begin{array}{l}+: 0,162 \\
-: \mathbf{0 , 1 7 2}\end{array}$ & & & $\begin{array}{l}+: \mathbf{0 , 4 7 1} \\
-: 0,101\end{array}$ \\
\hline & $4-N A K-I I$ & 27 & 39,4 & $\begin{array}{c}+: \mathbf{0 , 2 4 1} \\
-:: 0,0\end{array}$ & $\begin{array}{l}+\mathbf{0} \mathbf{0 , 2 3 6} \\
-: 0,225\end{array}$ & $\begin{array}{l}+: 0,22 \\
-: \mathbf{0 , 2 4 2}\end{array}$ & & & & & $\begin{array}{l}\text { +: } \mathbf{0 , 5 0 9} \\
\text {-: } 0,107\end{array}$ \\
\hline & $\begin{array}{c}2-N N N A K- \\
I I\end{array}$ & 41,8 & 24 & $\begin{array}{c}+: \mathbf{0 , 1 6 1} \\
-: 0,0208\end{array}$ & $\begin{array}{c}+: \mathbf{0 , 1 9 4} \\
-: 0,1\end{array}$ & $\begin{array}{l}+: \mathbf{0 , 1 4 9} \\
-: 0,145\end{array}$ & & $\begin{array}{c}+: \mathbf{0 , 1 7 4} \\
-: 0,12\end{array}$ & & & $\begin{array}{l}+: \mathbf{0 , 2 4 7} \\
-: 0,0471\end{array}$ \\
\hline
\end{tabular}

\section{AGRADECIMENTOS}

Os autores agradecem ao IME pela infraestrutura, e às agências de fomento CNPq (Processo 306156/2015-6) e FAPERJ (Grant no E-02/202.961/2017) pelo suporte financeiro. Este projeto também foi financiado pelo "excellence project FIM".

\section{MATERIAL SUPLEMENTAR}

Os produtos de degradação da DPA, 2-NDPA, EC e AK-II estão esquematizados no Material Suplementar, disponível de forma gratuita em http://quimicanova.sbq.org.br em formato PDF.

\section{REFERÊNCIAS}

1. Fryš, O.; Bajerová, P.; Eisner, A.; Skládal, J.; Ventura, K.; Propellants, Explos., Pyrotech. 2011, 36, 347.

2. Toxicological Profile for N-nitrosodiphenylamine, Agency for Toxic Substances and Disease Registry, 2017.

3. Drzyzga, O.; Jannsen, S.; Blotevogel, K. H.; Ecotoxicol. Environ. Saf. 1995, 31, 149.
4. Goodall, C. M.; Lijinsky, W.; Tomatis, L.; Wenyon, C. E. M.; Toxicol. Appl. Pharmacol. 1970, 17, 426.

5. Böhnlein-Mauß, J.; Kröber, H.; Propellants, Explos., Pyrotech. 2017, 42,54 .

6. Krumlinde, P.; Ek, S.; Tunestål, E. \& Hafstrand, A.; Propellants, Explos., Pyrotech. 2017, 42, 78 .

7. Fryš, O.; Bajerová, P.; Eisner, A. \& Skládal, J.; Cent. Eur. J. Energ. Mater. 2010, 7, 253.

8. Dobson, R.; Dejeaifve, A.; Monseur, L.; Fonder, N.; $7^{\text {th }}$ Nitrocelluose Symposium, Montreal, Canadá, 2016.

9. Magee, P. N.; Barnes, J. M.; Br. J. Cancer 1956, 10, 114.

10. Low, H.; Archives of Environmental Health - An International Journal 1974, 29, 256.

11. Araujo, M. E.; Cyrne, L.; Marinho, H. S.; Norberto, F.; Bol. Soc. Port. Quim. 2000, 79, 30.

12. Nieminuszczy, J.; Grzesiuk, E.; Acta Biochim. Pol. 2007, 54, 459.

13. Vollhardt, K. P. C.; Schore, N. E.; Organic Chemistry Structure and Function, $6^{\text {th }}$ ed., Bookman: Porto Alegre, 2013.

14. Drzyzga, O.; Chemosphere 2003, 53, 809.

15. Mestankova, H.; Schirmer, K.; Canonica, S.; von Gunten, U.; Water Res. 2014, 66, 399. 
16. Lussier, L. S.; Gagnon, H.; Bohn, M. A.; Propellants, Explos., Pyrotech 2000, 25, 117.

17. Helma, C.; Mol. Divers. 2006, 10, 147.

18. Maunz, A.; Gütlein, M.; Rautenberg, M.; Vorgrimmler, D.; Gebele, D.; Helma, C.; Front Pharmacol. 2013; 4, 1.

19. Willighagen, E. L.; Jeliazkova, N.; Hardy, B.; Grafström, R. C.; Spjuth, O.; BMC Res. Notes 2011, 4, 487.

20. Bohn, M. A.; J. Therm. Anal. Calorim. 2001, 65, 103.

21. Andrade, J.; Iha K, Rocco, J. A. F. F.; Franco, G. P.; Moreira, E. D.; Suárez-Iha, M. E. V.; Eclet. Quim. 2007, 32, 7.

22. Yucel, A.; Inal, E. K.; Akay, M. A.; Cent. Eur. J. Energy Mater. 2011, 8, 183.

23. Lussier, L. S.; Gagnon, H.; Bergeron, E.; Bohn, M. A.; Symp. Chem. Problems Connected Stab. Explos. 2004, 12, 183.

24. Lussier, L. S.; Bergeron, E.; Gagnon, H.; Propellants, Explos., Pyrotech. 2006, 31, 253.

25. Russom, C. L.; Bradbury, S. P.; Broderius, S. J.; Hammermeister, D. E.; Drummond, R. A.; Environ. Toxicol. Chem. 1997, 16, 948.

26. Antczak, P. Jo H. J; Woo, S.; Scanlan, L.; Poynton, H.; Loguinov. A.; Chan, S.; Falciani, F.; Vulpe, C.; Environ. Sci. Technol. 2013, 47, 11747.

27. Muehlbacher, M.; Spitzer, G. M.; Liedl, K. R.; Kornhuber, J.; J. Comput. Aided Mol. Des. 2011, 25, 1095.

28. Voltolini, A. M.; Horn, R. C.; Rocha, J. A. V.; Resumos da XIV Salão de Iniciação Científica - UFRGS, Porto Alegre, Brasil, 2002.

29. Swamidass, S. J.; Chen, J.; Bruand, J.; Phung, P.; Ralaivola, L.; Baldi, P.; Bioinformatics 2005, 21(Suppl. 1), i359.

30. Fligner, M. A.; Verducci, J. S.; Blower, P. E.; Technometrics 2002, 44, 110.

31. Gatnik, M. F.; Worth, A. P.; Review of software tools for toxicity prediction, available at https://publications.europa.eu/en/publicationdetail/-/publication/fb675f8d-8758-4451-83c6-638a153e1635/languageen, acessada em junho 2018.

32. Rybacka, A.; Rudén, C.; Andersson, P. L.; Basic Clin. Pharmacol. Toxicol. 2014, 115, 77.

33. Ruppenthal, J. E.; Toxicologia, Rede e-Tec-Brasil: Colégio Técnico Industrial de Santa Maria, Universidade Federal de Santa Maria: Santa Maria, 2013.

34. http://www.antares-life.eu/files/ANTARES_D14.pdf, acessada em junho 2018.
35. Baderna, D.; Golbamaki, N.; Maggioni, S.; Vaccari, M.; Colacci, A.; Benfenati, E. Em Global Risk-Based Management of Chemical Additives II; Baderna, D., ed.; Springer-Verlag: Berlin Heidelberg, 2012, p. 171.

36. http://always-on-target.com/wp-content/uploads/2017/07/5.56mm-CQTSDS.pdf, acessada em junho 2018.

37. https://echa.europa.eu/documents/10162/18584504/afa_dehp-0005-02aa_en.pdf/d355aac7-3d27-4f02-827f-bf105c272542, acessada em junho 2018.

38. DeGraeve, G. M.; Elder, R. G.; Woods, D. C.; Bergman, H. L.; Arch. Environ. Contam. Toxicol. 1982, 11, 487.

39. Bulich, A. A.; Greene, M. W.; Isenberg, D. L.; Aquatic Toxicology and Hazard Assessment 1981, 737, 338.

40. Huff, J. E.; Haseman, J. K.; DeMarini, D. M.; Eustis, S.; Maronpot, R. R.; Peters, A. C.; Persing, R. L.; Chrisp, C. E.; Jacobs, A. C.; Environ. Health Perspect. 1989, 82, 125.

41. Bingham, E.; Cohrssen B. Em Patty's Toxicology, 6 Volume Set; Bingham, E., Cohrssen B., eds;. John Wiley \& Sons: New Jersey, 2012.

42. Kalf, G. F.; Snyder, C. A.; Rev. Toxicol. 1987, 18, 141.

43. LeBlanc, G. A.; Bull. Environ. Contam. Toxicol. 1980, 24, 684.

44. La Voie, E. J.; Dolan, S.; Little, P.; Wang, C. X.; Sugie, S.; Rivenson, A.; Food Chem. Toxicol. 1988, 26, 625.

45. Toxicology and carcinogenesis studies of naphthalene (Cas $n^{\circ}$.91-20-3) in $f 344 / n$ rats., U. S. Department of Health and Human Services, 2000.

46. Narbonne, J.-F.; Cassand, P.; Alzieu, P.; Grolier, P.; Mrlina, G.; Calmon, J. P.; Mutat. Res. Lett. 1987, 191, 21.

47. Marchini, S.; Tosato, M. L.; Norberg-King, T. J.; Hammermeister, D. E.; Hoglund, M. D.; Environ. Toxicol. Chem. 1992, 11, 187.

48. Østergaard, G.; Em The Nordic Expert Group for Criteria Documentation of Health Risks from Chemicals: I25. Toluene, Marklund, S., ed.; National Institute for Working Life: Stockholm, 2000.

49. Mohtashamipur, E., Norpoth, K., Woelke, U.; Huber, P.; Arch. Toxicol. 1985, 58, 106 .

50. https://ntp.niehs.nih.gov/ntp/htdocs/lt_rpts/tr203.pdf, acessada em junho 2018.

51. Nomiyama, K.; Minai, M.; Suzuki, T.; Kita, H.; Ind. Health 1967, 5, 143. 